\title{
DEFORMATION FEATURES OF UNIAXIAL COMPRESSION OF SAMPLES FROM THE POTATO TUBERS FLESH
}

\author{
Valeriy G. Zhukov*, Nikolay D. Lukin \\ All-Russian research Institute for Starch Products - Branch of V.M. Gorbatov Federal Research Center for Food Systems of RAS, \\ Kraskovo, Moscow Region, Russia
}

\section{KEYWORDS:}

potato, flesh, uniaxial compression, stress, strain, destruction, diagram, elastic, brittle

\begin{abstract}
The article analyzes the deformation mechanism under uniaxial compression of cylindrical samples cut from the pulp of potato tuber. In its typical S-shaped stress-strain diagram, three characteristic zones of different types of dominant deformation can be distinguished. Clarified the boundary between the second and third zones. The rationale for changing the type of deformations in each zone by adding a new type to the previous one is presented. Grounded elastic deformation in the first zone, which is associated with stretching of the cell membranes. In the second zone, the destruction of individual cells distributed in the sample volume occurs and in the third zone - the predominant gradual unification of these destructions. An explanation is given of the characteristic brittle fracture of the sample with significant residual deformations at the end of the third zone. The erroneous determination of the deformations of the second and third zones as plastic (yield) was noted. In this regard, a new term was introduced - modulus of rigidity $\mathrm{Z}$. The change in the modulus of stiffness $\mathrm{Z}$ with increasing irreversible deformations indicates structural changes in the flesh of the sample due to its gradually collapsing cell structure. The reason for straightening diagrams for samples from fresh, hard potato tubers is explained. Taking into account the type of deformations by zones allows us to reasonably proceed to the creation of criteria for quantitative assessments of the degree of flaccidity of potato tubers.
\end{abstract}

\section{Introduction}

The basis of analytical calculations and design of processes and equipment for the mechanical processing of plant biomaterials, including for potatoes, and animal origin are stress-strain studies [1]. Often used for this purpose the test for the destruction at the conditions of uniaxial compression of carved from their flesh cylindrical samples is informative and universal method $[2,3,4]$. Such studies establish the nominal compression stresses (hereinafter - stresses), including the ultimate compressive stress $\sigma_{U}$ (compressive strength), the relative deformation $\varepsilon$ (hereinafter - strain) and the general form of the fracture graph in the stress-strain $(\sigma-\varepsilon)$ coordinates [5]. With a great attention of the researchers to obtaining diagrams of destruction by uniaxial compression of samples of various plant products, the analys of the deformation was investigated only in the most general form and mostly limited to identifying of conditions the absence of bruising at low loads for example, in potatoes [6], apples and pears [7,8] and by registration of destroyed cells of apple flesh [9].

This article is devoted to the analysis of the type of deformation under uniaxial compression of cylindrical samples cut from the flesh of the potato tubers.

According to known publications of the results of research on the physic-mechanical properties of fruits and vegetables and own experiments $[3,10,11,12]$, a number of essential features of the compression diagrams of cylindrical samples cut from their flesh have been revealed. So, for potatoes, along with the manifestation of the relaxation properties of its flesh [11]. The diagram of the destruction by compression of samples from fruits and vegetables of long-term storage has an S-shape [13,14,15]. In samples from fresh potato tubers and at a high loading rate, the diagram is straightened, approaching a straight line $[2,8,16]$. The articles $[11,15,16]$ emphasized that the destruction of potato samples occurs along a plane inclined at an angle of $45^{\circ}$ to the axis of the sample, that is, according to the type of brittle fracture.

An important observation is also the fact that the elastic strain of potato samples occurs only in a small initial zone of the stress diagram in the region up to $\sigma \approx 0.1 \mathrm{MPa}$. It can be as low as $20 \%$ of the ultimate strength $\sigma \mathrm{U}$. This characteristic stress is denoted below as $\sigma_{0,1}=\sigma_{B}=0.1 \mathrm{MPa}$ was noted in the article [17]. In it, the diagram was conditionally divided into two zones: up to stress $\sigma \approx 0.1 \mathrm{MPa}$, called quasi-elastic, and above, called yield zone.

In accordance with this dichotomy, for the first zone, author applied the traditional notion of elastic modulus $(E)$, and for the other, the notion of yield modulus $\left(E_{w}\right)$. However, the reason for introducing the concepts of quasi-elasticity and yield was not explained. As our experiments showed [12,15], one cannot agree with this interpretation of the diagram zones, since the first zone reflects the traditional notion of elasticity, non-linear in this case, and the second does not reflect the reasons for the appearance of residual straines and the change of their formes which are associated with the notion of brittle material. Further loading $(\sigma>0.1 \mathrm{MPa})$ leads to intensely increasing inelastic strain up to splitting with $\varepsilon_{S} \approx 0.3$ and even more $[2,8,17]$. In this regard, article [15] concluded that the tangent of the angle of inclination to the axis of strain of linear approximations of a diagram or its parts, including elastic and inelastic components, should not be called the Young's modulus, i.e. modulus of elasticity. It is proposed to call it the rigidity modulus, and denoted as $Z$.

\section{Materials and methods}

The article is based on the results of experiments with cylindrical samples cut from potato tubers. For objectivity, the results were analyzed of the articles of both our own and other authors. The experiments were carried out on samples that had a ratio of length to diameter from 1 to 2 . The greater this ratio, the clearer the character of the brittle destruction of the sample. Fresh, hard tubers showed a diagram that was close to straight. In flabby tubers that have been stored for a long time, the diagram had a characteristic S-shape. In the publications mentioned, the methods of conducting experiments and obtaining diagrams were different: from building diagrams by tables of results to automated ones using computer programs. In the presented article there are links to all borrowings.

Cylindrical samples in our articles had a ratio between the length and diameter of the sample $l_{0} / d_{0}=25 / 14=1,79$. They 
remained steadiness in compression tests, although in the literature on the properties of fruits and vegetables, the magnitude of this ratio is usually limited to one.

\section{Results and discussion}

On a typical S-shaped diagramm of fracture by uniaxial compression of a cylindrical sample cuted from a flabby potato tuber (Figure 1), it is actually necessary to distinguish three characteristic zones of the predominant type of deformations.

First $(A B)$ - elastic deformation at stresses from zero to $\sigma_{B}=0.1 \mathrm{MPa}$. The second $(B C)-$ an addition to the elastic deformation of inelastic deformation at the initial fragmented destruction of cells with the least durable and most loaded shells in the stress range from $\sigma_{B}$ to $\sigma_{C}$. The third $(C P)$ is the predominant inelastic deformation under the conditions of gradual unification of separate fractures at stresses from $\sigma_{C}$ to $\sigma_{U}$, ending with brittle fracture of the sample. The selection on the diagram of the three zones was first shown in the article [12]. Our article contains a number of important additions and clarifications.

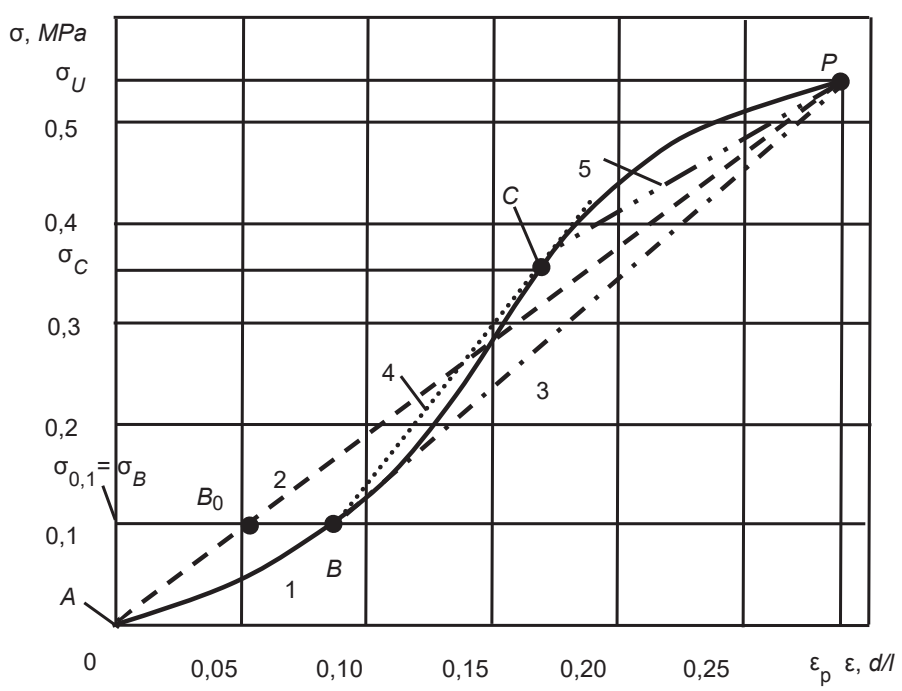

Figure 1. A characteristic stress-strain diagram of the fracture by uniaxial compression of a cylindrical specimen cut from a flabby potato tuber: $\sigma-$ compressive stress, $\mathrm{MPa} ; \varepsilon-$ strain, $d / l$ (dimentionless); continuous line $1-$ typical experimental $S$-shaped diagram of compression of a cylindrical sample from a flabby tuber; the idealized linear approximations of the diagram and its individual zones correspond to: the dash line $2-$ fresh tuber for the interval $0-\sigma_{U}$, the dash-dotted line $3-$ for the interval $\sigma_{0,1}-\sigma_{U}$, the dotted $4-$ for the interval $\sigma_{0,1}-\sigma_{C}$, the dash-two-dotted 5 - for interval $\sigma_{C}-\sigma_{U} ; B, B_{0}, C, P$ - characteristic points; $P$ - the point of the sample brittle fracture; characteristic stresses of the sample: $\sigma_{0,1}=\sigma_{B}=0.1 \mathrm{MPa}, \sigma_{U}-$ the ultimate strength, $\sigma_{C}$ - the stress at point $C$ corresponding to the change in the curvature of the line of the diagram from concave to convex; $\varepsilon_{p}-$ the strain of sample brittle fracture at the point $P$

The shape of the first zone of the diagram is affected by the destroyed surface cells of two flat transverse end and cylindrical surfaces of the sample cut from the tuber, and also the stress difference between the intact cells of the flesh. The cell membranes are stretched elastically and the stresses are leveled across the entire volume of the sample.

The $A B$ segment of diagram is non-linear for a sluggish and close to linear $\left(A B_{0}\right)$ for a fresh tuber $[12,15,16]$. Only minor residual deformations appear in this zone. The elastic nature of the deformation at $\sigma<0.1 \mathrm{MPa}$ is confirmed by experiments with apples and pears $[7,8]$. The author of these articles notes the absence of bruises in the zone of low load. For this reason, the first zone shows no noticeable residual deformations and is called the elastic deformation zone. Bruises appear only when the cell structure of the flesh is destroyed, that is, at high stresses, in the second and third zones.

The noted features are also characteristic for samples cut from other plant materials and materials of animal origin that have a cellular structure [18].

From point $B$, the second zone begins. It reflects the continuation of the growth of elastic strains, the appearance and growth of inelastic strains due to the destruction membrans of individual cells throughout the sample volume. The continuation of the growth of elastic strains is confirmed by an increase in the range of residual strains at intermediate unloadings, shown in $[8,12]$. The gradual accumulation of damage of the overloaded and least durable individual cells, scattered by volume, should be considered to volumetric micro-destruction. At the same time, the rigidity of the sample increases due to an increase in stresses in intact membranes of cells.

For ductile materials, the modulus of elasticity remains almost constant until destruction $(E=$ const) [5], which indicates their unchanged structure throughout the diagram. In the samples cut from the flesh of potato tuber, a gradual change in the modulus of rigidity $Z$ with increasing irreversible deformations is observed, which confirms the structural damage in it flesh [12]. These fractures are essentially similar to the appearance of microcracks in brittle structural materials.

The third zone begins at point $C$ with a change in the curvature of the $S$-shaped diagram from concavity to convexity. The third zone reflects the continuation, of the destruction of individual cells and a gradual fusion of their volumes, which corresponds to the concept of macrodestruction. Such a form of destruction is reflected in the photographs in the work [9]. This means that the forms of changes in the cell structure of the flesh of the tuber, which began in the previous zones, are increasing, and new ones are added to them. Based on this, it is possible to assert a priori that biological materials with a cellular structure will fracture like a fragile body. Hence, the term plasticity often applied to them does not correspond to the essence of irreversible deformations occurring in them. Gradually the process of merging the volumes of destroyed cells goes into a predominant one. The number of unexploded cells remains ever less, and the rigidity of the sample with a significant share of macro-destructions is markedly reduced. As a result, its strain grow more intensively with a smaller increase in load, and the diagram becomes more and more gently sloping. The second and third zones can merge into a single one, close to linear (line 3) in conditions sufficiently fast loading due to damping by relaxation features of the flesh cells. Tests of samples from fresh potatoes (with a high turgor value) give a diagram of all three zones close to the idealized linear (straight line $A P-$ line 2). The third zone ends with the destruction of the sample by splitting along a plane inclined at an angle of $45^{\circ}$ to axle of longitudinal load, i.e. on a brittle type, confirming the description of processes in the second and third zones.

In the process of compression, a certain thickening of the sample occurs. However, in the second and third zones, the actual number of intact cells that provide the strength of the sample decreases to a greater extent, i.e. the area formed by them decreases in cross section. Hence, the actual (true) stress and ultimate stress, counted as the ratio of the applied force to the area occupied by the intact cells in the cross section, is in fact greater than the nominal. Such more accurate value is introduced in some cases for plastic structural materials, when the true cross-sectional area of the samples is taken into account by dividing the force by the area of the narrowing when stretched or thickening under compression [19]. 
The high value of turgor provides a more uniform initial tension in the cell membrane. Consequently, the nature of their destruction is the same and occurs with constant intensity throughout the diagram. Therefore, the diagram view approaches the straight line 2, although at its beginning some nonlinearity may appear $[2,12,16]$. However, in any form, the first zone also ends at $\sigma_{0,1}=0.1 \mathrm{MPa}$ at point $B_{0}$, after which inelastic deformations begin to grow noticeably.

The revealed change of the deformation mechanism by zones allows one to proceed to the creation of criteria for quantitative assessments of the flaccidity degree of potato tubers.

\section{Conclusion}

$S$-shaped diagrams of destruction by uniaxial compression of samples cut from the flesh of flaccid potato tubers clearly show three characteristic zones with differing deformation mechanisms.
The boundaries of the zones are: between the first and second - the stress $\sigma_{0,1}=0.1 \mathrm{MPa}$ (point $B$ ), between the second and third - the change in the curvature of the $S$-shaped diagram from concavity to convexity at point $C$.

Elastic deformations of the first zone are supplemented in the second by microdestruction of individual cells and in the third by the union of groups destructed of cells, which can be characterized macrodestruction. Thus, the deformations of the previous zone do not disappear, but are complemented by a new type in the next zone. The third zone ends with a brittle fracture of the sample with significant residual deformation. Tests of samples from fresh potatoes (with a high turgor value) give a diagram of all three zones close to a single idealized linear one. At the same time, the boundary between the second and third zones will be disappear, but on the whole, the pattern of deformation and destruction of the cell structure of the potato tubers revealed by the zones remains.

\section{REFERENCES}

1. Andreev, N.R. (1980). Research and development of potato impact crusher for starch production. Author's abstract of the dissertation for the scientific degree of Candidate of Technical Sciences. Moscow, MTIMMP, 26 p. (in Russian).

2. Abbott, I.A. (1999). Ouality measurement of fruits and vegetables. Postharvest Biology and Technology, 15(3), 207-225. DOI: 10.1016/S09255214(98)00086-6

3. Bentini, M., Caprara, C., Martelli, R.. (2009). Physico-mechanical properties of potato tubers during cold storage. Biosystems Engineering, 104(1), 25-32. DOI: 10.1016/j.biosystemseng.2009.03.007

4. Canet, W., Alvarez, M.D., Gil, M J. (2007). Fracture behaviour of potato samples (cv. Desiree) under uniaxial compression. Journal of Food Engineering, 82(4), 427-435. DOI:10.1016/j.jfoodeng.2007.02.054

5. Beer, F.P., Russell, J.Jr., Dewolf, J.T., Mazurek, D.F. (2012). Mechanics of Materials. Sixth edition. Published by McGraw Hill. Science.- 388 p. ISBN00733802889780073380285.

6. Azam, M.M., Essia, A.H.A. (2015). Comprehensive Evaluation of Dynamic Impact as a Measure of Pjnfnj Quality. International Journal of Food Engineering and Technology, 1(1), 1-10.

7. Blahovec, J. (2001). Static mechanics and texture of fruits and vegetables. Research in Agricultural Engineering, 47(4), 144-169.

8. Blahovec, J., Vlckova, M., Paprstein, F. (2002). Static low-level bruising in pears. Research in Agricultural Engineering, 48(2), 41-46.

9. Shah, L.B.M. (2016). Instrumental and Ultrasonic Techniques in Quality Evaluation of Fresh Fruit and Vegetables. Submitted in accordance with the requirements for the degree of Doctor of Philosophy. The University of Leeds. School of Food Science and Nutrition.

10. Paoletti, F., Moneta, E., Bertone, A., Sinesio, F. (1993). Mechanical Properties and Sensory Evaluation of Selected Apple Cultivars. LWT - Food Science and Technology, 26(3), 264-270. DOI: 10.1006/fstl.1993.1055
11. Zhukov, V.G., Andreev, N.R., Bezrukov, D.V. (2016). Relaxation Effect at Testing Potato Samples by Compression. Achievements of Science and Technology of AIC, 30(11), 121-122. (in Russian)

12. Zhukov, V.G., Andreev, N.R., Lukin, N.D. (2017). Deformation Mechanism by Compression of Cylindrical Samples from Potato Tubers. Achievements of Science and Technology of AIC, 31(6), 80-83. (in Russian)

13. Laza, M. (1999). Mechanical properties affecting slicing performance of potatoes. Thesis Submitted to the Facuity of Graduate Studies In Partial Fulfillment of the Requirements for the Degrtt of Master of Science. Food Science Departmerit University of Manitoba, Winnipeg, Manitoba.

14. Oey, M.L., Vanstreels, E., De Baerdemaeker, J., Tijskens, E., Ramon, H., Hertog, M.L.A.T.M., Nicolaï, B. (2007). Effect of turgor on micromechanical and structural properties of apple tissue: A quantitative analysis. Postharvest Biology and Technology, 44 (3), 240-247. DOI:10.1016/j.postharvbio.2006.12.015

15. Zhukov, V.G., Andreev, N.R., Lukin, N.D. (2019). Peculiarities of potato flesh sample deformation by uniaxial compression. Norwegian Journal of development of the International Science, 26(2), 3-5.

16. Savrasova, N.R. (2012). Results of experimental definition of the module of elasticity and strength of a tuber of a potato. VESTNIC ChSAA, 60, 80-82. (in Russian)

17. Blahovec, Jiř́, Sobotka, J. (2007). Potato tuber permittivity during deformation in compression. Research in Agricultural Engineering, 53(3), 79-84. DOI:10.17221/2124-rae

18. Chizhikova, T.V. (1982) Machines for grinding meat and meat products M, Light and food industry.- 302. (in Russian)

19. Zhukov, V.G. (2012). Mechanics. Strength of materials. Krasnodar-S-PbM., Lan.-414 p. ISBN978-5-8114-1244-0 (in Russian)

\section{AUTHOR INFORMATION}

Valery G. Zhukov - doctor of technical sciences, Chief Researcher, All-Russian Research Institute for Starch Products - Branch of V.M. Gorbatov Federal Research Center for Food Systems of RAS, 140051, Moscow region, Kraskovo, ul. Nekrasov, 11, Tel.: +7-495-557-15-00, e-mail: vniik@arrisp.ru *corresponding author

Nikolay D. Lukin - doctor of technical sciences, Acting Director, All-Russian Research Institute for Starch Products - Branch of V.M. Gorbatov Federal Research Center for Food Systems of RAS, 140051, Moscow region, Kraskovo, ul. Nekrasov, 11, Tel.: +7-495-557-15-00, e-mail: vniik@arrisp.ru Authors are equally relevant to the writing of the manuscript, and equally responsible for plagiarism

The authors declare no conflict of interest

Received 16.04.2019 Accepted in revised 14.05.2019 Accepted for publication 10.06.2019 